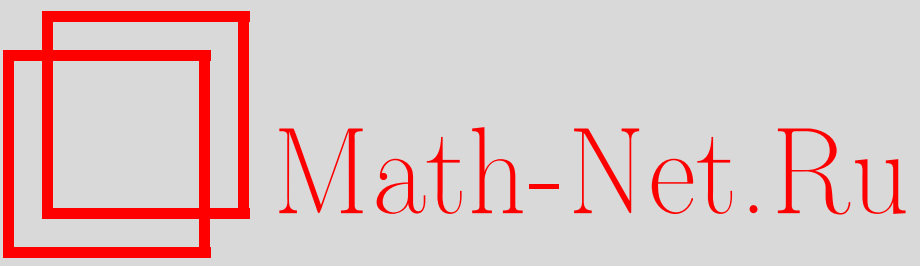

Ю. Л. Болотин, Ю. П. Вирченко, Статистика квазиэнергий квантовомеханических систем для регулярного и хаотического режимов с гамильтонианами, периодически изменяющимися во времени, ТМФ, 1996, том 108, номер 3, 431-447

DOI: https://doi.org/10.4213/tmf1201

Использование Общероссийского математического портала Math-Net.Ru подразумевает, что вы прочитали и согласны с пользовательским соглашением

http://www.mathnet.ru/rus/agreement

Параметры загрузки:

IP : 54.162 .127 .20

26 апреля 2023 г., 10:24:15 
ТЕОРЕТИЧЕСКАЯ

И МАТЕМАТИЧЕСКАЯ

ФИЗИКА

Том 108, № 3

сентябрь, 1996

\author{
Ю. Л. Болотин, Ю. П. Вирченко
}

\title{
СТАТИСТИКА КВАЗИЭНЕРГИЙ КВАНТОВО-МЕХАНИЧЕСКИХ СИСТЕМ ДЛЯ РЕГУЛЯРНОГО И ХАОТИЧЕСКОГО РЕЖИМОВ С ГАМИЛЬТОНИАНАМИ, ПЕРИОДИЧЕСКИ ИЗМЕНЯЮШИМИСЯ ВО ВРЕМЕНИ
}

Рассматриваются квантово-механические системы с гамильтонианами, периодически изменяющимися во времени. В предположении, что спектр оператора Флоке дискретный и расстояния между квазиэнергиями допускают статистическое описание на основе непрерывной плотности распределения, показано, что плотность индуцированного распределения вероятностей для расстояний между подходящим образом ренормированными дробными частями квазиэнергий, определенных по $\bmod (\hbar \omega)$, сколь угодно близка к экспоненциальной плотности распределения, если число уровней неограниченно возрастает. Этот результат не зависит от исходного закона распределения. Предложен альтернативный метод статистического описания дробных частей, который позволяет различать исходные статистические законы распределения квазиэнергий для регулярного и хаотического режимов.

\section{1. ВВЕДЕНИЕ}

В этой работе мы рассматриваем возможности численного статистического исследования спектров квазиэнергий квантово-механических систем с гамильтонианами $H(\omega t)$, периодически зависяшими от времени $t(\omega=2 \pi / T, T$-период изменения). Такое исследование обычно выполняется в том числе, если рассматриваемая система не поддается аналитическому изучению. В работах, относяшихся к этому направлению (см., например, библиографию в [1]), не рассматривался случай, когда $\omega$ имеет порядок характерной внутренней частоты $\nu$ соответствуюшей невозмушенной системы в отсутствие периодической зависимости гамильтониана. Это связано с техническими трудностями (см. раздел 2). При исследовании этого случая вместо статистического описания собственно квазиэнергий удобно описывать статистические свойства спектров в терминах плотности распределения расстояний между дробными частями квазиэнергий, определенных по $\bmod (\hbar \omega)$. Однако поскольку среднее значение этих расстояний стремится к нулю, необходимо построить статистику соответствуюших ренормированных величин, чтобы получить для них некоторый предельный статистический закон при числе $N$ уровней квазиэнергий, стремяшемся к бесконечности. Единственно возможный способ перенормировки в этом случае состоит в растяжении порядка $N$ расстояний между дробными частями. Мы покажем в этой статье, что при некоторых предположениях 
относительно общей структуры спектра соответствующий предельный закон является универсальным, а именно экспоненциальным. Этот результат не позволяет различать регулярный и хаотический режимы посредством изучения численно построенных гистограмм при достаточно больших $N$, если гистограммы получаются на основе указанной выше ренормализационной процедуры. В связи с этим мы предлагаем другой метод статистического исследования. Он основан на изучении предельной функции распределения расстояний между дробными частями квазиэнергий, определенных по $\bmod (\hbar \omega)$. Ренормализационная процедура в этом случае состоит в возведении функции распределения в степень $N^{-1}$.

Далее в разделе 2 мы обсудим качественно постановку задачи. В разделе 3 вводится вероятностная модель для статистического описания дробных частей квазиэнергий. В разделах 4 и 5 мы доказываем основную асимптотическую формулу и теорему универсальности, а в разделе 6 указываем на возможность альтернативного метода статистического исследования дробных частей квазиэнергий.

\section{2. ПОСТАНОВКА ЗАДАЧИ}

Для любой квантово-механической системы с гамильтонианом $H(\omega t)$, периодически изменяющимся во времени, на основе преобразования Флоке-Ляпунова [2-7] всегда можно ввести соответствуюшую автономную систему с гамильтонианом $H$. Спектр $\left\{\epsilon_{n}\right\}$ гамильтониана $H$ называется спектром квазиэнергий исходной системы. Гамильтониан $H$ не определен единственным образом. Эта неоднозначность такова, что квазиэнергии определены по $\bmod (\hbar \omega)$. Неоднозначность исчезает, если потребовать непрерывной зависимости квазиэнергий от некоторого параметра $\lambda$, который вводит в гамильтониан $H_{\lambda=0}$ периодическую зависимость. В этом случае $H$ определяется однозначно для $\lambda \neq 0$, т.к. $H_{\lambda=0}$ является гамильтонианом автономной системы. Далее мы считаем, что квазиэнергии $\epsilon_{n}(\lambda)$ построены путем такого $\lambda$-продолжения. Непрерывность по $\lambda$ периодически изменяюшегося возмушения позволяет придать физический смысл квазиэнергиям. В то же время эта непрерывность приводит к техническим трудностям при вычислении квазиэнергий для немалых значений $\lambda$. В этом случае, если мы хотим численно определить при фиксированном $\lambda$ часть спектра, содержащуюся в некотором заданном интервале энергий, то для этого необходимо определить более широкую часть спектра для всех промежуточных значений $\lambda$, лежаших между нулем и данным значением. Это необходимо проделать пошагово с достаточно малым шагом, для того чтобы численно построить продолжение квазиэнергий $\epsilon_{n}(\lambda)$ в виде непрерывных функций $\lambda$.

Спектр мультипликаторов $\left\{\exp \left(-i T \epsilon_{n} / \hbar\right)\right\}$ (спектр оператора Флоке), в отличие от спектра квазиэнергий $\epsilon_{n}$, определяется единственным образом. Указанная численная процедура определяет целое число $\hbar \omega$-оборотов, на которое изменяются фазы мультипликаторов при возрастании $\lambda$ вплоть до данного ее значения. Заметим, что спектр мультипликаторов во многих случаях плотно покрывает единичную окружность и не имеет абсолютно непрерывной части. Одним из таких случаев является, например, одномерное движение частицы в прямоугольной потенциальной яме. Вследствие этого имеется проблема выделения множества мультипликаторов, которые соответствуют собственным значениям оператора $H$, содержашимся в некотором фиксированном энергетическом интервале. Указанное $\lambda$-продолжение осушествляет такое выделение разреженного множества квазиэнергий и определяет на нем порядок. 
Для того чтобы избежать описанной выше сложной процедуры вычисления квазиэнергий, обычно рассматриваются малые значения $\lambda$. В этом случае, если энергетический интервал с размером $\hbar \omega$ содержит большое число уровней, то в этом интервале имеется много квазиэнергий $\epsilon_{n}(\lambda)$ с одной и той же целой частью величины $\left(\epsilon_{n}(\lambda) / \hbar \omega\right)$, и эта целая часть имеет значение, совпадающее со значением при $\lambda=0$. Поэтому $\lambda$-продолжение тривиально, и упорядоченность дробных частей $(\epsilon(\lambda) / \hbar \omega) \bmod 1$ не изменяется. Последнее свойство является очень важным для построения процедуры численной диагонализации оператора Флоке, при которой мультипликаторы, соответствующие рассматриваемому множеству квазиэнергий $\epsilon_{n}(\lambda)$, вычисляются наиболее точно. Большое число уровней гарантирует, что мы имеем для них достаточно убедительную статистику, т.е. возможно статистическое описание.

Пусть $\nu$ - некоторая характерная частота невозмущенной системы. Для того чтобы удовлетворить условию большой выборки квазиэнергий, необходимо, чтобы среднее расстояние $\hbar \nu$ между квазиэнергиями было значительно меньше $\hbar \omega$, т.е. должно вьполняться неравенство $\nu \ll \omega$. Для малых значений $\lambda$ частота $\nu$ изменяется незначительно и неравенство не нарушается при изменении $\lambda$. Рассматриваемые квазиэнергии смешаются на величины, которые намного меньше $\nu$, и поэтому почти все они не покидают данный энергетический интервал с размером $\hbar \omega$. Таким образом, введение статистического набора для квазиэнергий без численного построения функций $\epsilon_{n}(\lambda)$ для малых значений $\lambda$ оправданно.

Если условие $\nu \ll \omega$ нарушается, то возникают численные трудности при статистическом изучении спектров квазиэнергий для малых $\omega$. В этом случае необходимо решить довольно громоздкую численную задачу реконструкции истинных фаз для очень большого числа мультипликаторов. С другой стороны, статистическое изучение является очень важным, т.к. можно ожидать, что спектры квазиэнергий проявляют универсальные статистические свойства, как это имеет место для спектров энергий автономных квантовых систем. Это явление состоит в том, что существуют универсальные предельные законы распределения, описывающие статистику расстояний между уровнями, когда число уровней в фиксированном энергетическом интервале неограниченно возрастает. (Число уровней управляется путем изменения $\hbar[1,7-10]$.$) Эти законы за-$ висят только от поведения предельных классических систем при $\hbar \rightarrow 0$, т.е. от того, является ли их движение регулярным или хаотическим. Так как классические гамильтоновы системы с гамильтонианами, периодически зависящими от времени, также могут проявлять как регулярный, так и хаотический характер эволюции [11], то можно ожидать сушествования аналогичной универсальности статистики квазиэнергий в соответствуюших квантовых системах; и тип закона распределения, описываюшего статистику, должен зависеть, по-видимому, только от качественных свойств поведения предельной классической системы. Таким образом, проверка этой гипотезы требует возможности набирать гистограммы расстояний между квазиэнергиями для некоторого широкого набора энергий и частот $\omega$.

Естественно возникает следующий вопрос: можно ли изучать проблему универсальности только на основе статистики мультипликаторов? Мы даем ответ на этот вопрос в настоящей работе. Во-первых, мы доказываем, что статистика расстояний между $\hbar \omega$-дробными частями квазиэнергий описывается универсальным экспоненциальным законом при $N \rightarrow \infty$, если расстояния ренормируются посредством растяжения порядка $N$. Таким образом, мы получаем на этом пути универсальный закон распределения для дробных частей, но он не позволяет различать регулярный и хаотический режимы.

4 Теоретическая и математическая физика, т. 108, № 3, 1996 г. 
Во-вторых, мы рассматриваем другую возможность получения предельных статистических законов, когда функция распределения возводится в степень $N^{-1}$. Предельная функция распределения в этом случае зависит от исходной плотности распределения расстояний между квазиэнергиями. Прямое вычисление показывает, что предельная функция распределения является линейной для экспоненциальной исходной плотности распределения и нелинейной в случае общего положения. В частности, она является нелинейной для вигнеровской исходной плотности распределения.

Результаты, полученные ниже, основаны на следующих предположениях о структуре спектра квазиэнергий:

1) спектр оператора Флоке не имеет абсолютно непрерывной части;

2) спектр квазиэнергий является настолько сложным, что возможно его статистическое описание;

3) случайные расстояния между уровнями статистически независимы в рамках статистического описания.

\section{3. ВЕРОЯТНОСТНАЯ МОДЕЛЬ}

В этом разделе мы будем изучать статистику дробных частей квазиэнергий с помощью вероятностных методов на основе предложений 1-3. Такой подход особенно удобен в случае, если условие $\nu \ll \omega$ нарушается и имеется только небольшое число уровней квазиэнергий в энергетическом интервале с шириной $\hbar \omega$, недостаточное для убедительной статистики. Напротив, малое число уровней в интервале $\hbar \omega$ дает возможность изучать статистику дробных частей квазиэнергий, определенных по $\bmod (\hbar \omega)$ с помощью вероятностных методов, т.к. дробные части быстро "забывают" абсолютные значения фазы, когда число уровней возрастает. Дробные части в этом случае образуют выборку, плотно покрьвающую интервал с размером $\hbar \omega$.

Мы считаем далее, что спектр квазиэнергий настолько сложен, что мы можем применить вероятностный подход для его изучения. В рамках этого подхода мы считаем, что расстояния между уровнями фиксированной изучаемой системы являются квазислучайными, и поэтому мы вводим плотность вероятности для описания их статистики. Тогда мы можем поставить задачу о вероятности нахождения ближайшего уровня к данному случайно выбранному. Такой подход является распространенным, когда изучаются сложные энергетические спектры автономных систем [12]. Далее мы предполагаем согласно разделу 2, что:

1) множество $\left\{\epsilon_{n} ; n=1,2, \ldots\right\}$ является дискретным спектром квазиэнергий, занумерованных в порядке возрастания;

$2)$ расстояния между уровнями $\left(\epsilon_{n+1}-\epsilon_{n}\right)=\hbar \omega \Delta_{n}-$ случайные, одинаково распределенные величины с непрерывной плотностью распределения $f($.) такой, что $f(\Delta)=0$ для $\Delta<0$ (статистическая эквивалентность не имеет места для энергетического интервала, где имеются как регулярный, так и хаотический режимы эволюции);

$3)$ случайные величины $\left\{\Delta_{n} ; n=1,2, \ldots\right\}$ статистически независимы.

Постулаты 1-3 определяют “случайную очередь" [13] как математически адекватную модель для статистического описания сложных спектров квазиэнергий. Наша цель - исследовать статистические закономерности случайных величин $\epsilon_{n} \bmod (\hbar \omega)$ в рамках этой модели. Далее удобно рассмотреть случайные переменные $\zeta_{n}=\left(\epsilon_{n} / \hbar \omega\right) \bmod 1$ и $\xi_{n}=\Delta_{n} \bmod 1, n=1,2, \ldots$, для которых имеем

$$
\zeta_{n}=\left(\Delta_{1}+\Delta_{2}+\cdots+\Delta_{n-1}+\epsilon_{1} / \hbar \omega\right) \bmod 1
$$


И

$$
\zeta_{n}=\left(\zeta_{n-1}+\xi_{n-1}\right) \bmod 1
$$

вследствие тождества $(x+y) \bmod 1=(x \bmod 1+y) \bmod 1$. Случайные переменные $\xi_{n}, n=1,2, \ldots$, статистически независимы и одинаково распределены с плотностью

$$
g(\xi)=\sum_{n=0}^{\infty} f(\xi+n), \quad \xi \in[0,1]
$$

т.к. все случайные значения $(\xi+n), n=0,1,2, \ldots$, переменной $\Delta$ приводят к одному и тому же случайному значению $\xi$. Случайные переменные $\zeta_{n-1}$ и $\xi_{n-1}, n=2,3, \ldots$, в (3.2) статистически независимы, что следует из их определения. Тогда случайная последовательность $\left\{\zeta_{n} ; n=1,2,3, \ldots\right\}$ образует марковскую цепь с фазовым пространством $[0,1)$ и плотностью условной вероятности перехода $g(x, y), x, y \in[0,1)$, т.к. случайная переменная $\zeta_{n}$ не зависит от предыдущих величин $\zeta_{1}, \ldots, \zeta_{n-2}$, когда $\zeta_{n-1}=y$ фиксирована. Легко найти явный вид функции $g(x, y)$ на основе $(3.1)$, т.к. имеет место соотношение $\zeta_{n}=\left\{\Delta_{n-1}+\zeta_{n-1}\right\}$. Из определения функции $g(x, y)$ имеем

$$
\begin{aligned}
g(x, y) d x & =\mathbb{P}\left\{\zeta_{n} \in[x, x+d x) \mid \zeta_{n-1}=y\right\}= \\
& =\mathbb{P}\left\{\left(\Delta_{n-1}+y\right) \bmod 1 \in[x, x+d x)\right\}=\sum_{n=0}^{\infty} f(x-y+n) d x
\end{aligned}
$$

и поэтому

$$
g(x, y)=g((x-y) \bmod 1)
$$

т.к. для $x<y$ суммирование в (3.4) начинается с $n=1$. Таким образом, дробные части $\zeta_{1}, \zeta_{2}, \ldots, \zeta_{n}, \ldots$ образуют траекторию марковской цепи, и поэтому плотность вероятности $g_{N}(x)$ для дробной части $N$-го уровня определяется выражением

$$
g_{N}(x)=\left(G^{N-1}\right)\left(x, \epsilon_{1} / \hbar \omega\right)
$$

где $G$ - интегральный оператор с ядром $g(x, y)$,

$$
g_{N}(x)=\int_{0}^{1} g(x, y) g_{N-1}(y) d y
$$

ТЕОРЕМА 1. Марковская цепь с плотностью условной вероятности перехода $g(x, y)$ имеет единственную неподвижную плотность $g_{\infty}(x)=1$, которая является притягивающей неподвижной точкой при $N \rightarrow \infty$ в пространстве $C(0,1)$ непрерывных функиий на $[0,1]$.

ДокАЗАТЕльство. Рассмотрим последовательность $\left(G^{N} p\right)(x), N=1,2, \ldots$, где $p(x)$ - произвольная функция в $C(0,1)$. Рассматриваемые функции полностью характеризуются коэффициентами Фурье

$$
c_{l}(N)=\int_{0}^{1} \exp (2 \pi i l x)\left(G^{N} p\right)(x) d x, \quad l \in \mathbf{N}
$$


для каждого $N=1,2, \ldots$ Вычисляя коэффиициенты Фурье $c_{l}[G p]$ преобразования $(G p)$, имеем

$$
\begin{aligned}
c_{l}[G p] & =\int_{0}^{1} \exp (2 \pi i l x)(G p)(x) d x= \\
& =\int_{0}^{1} \exp (2 \pi i l x) d x \sum_{n=0}^{\infty} \int_{0}^{1} f(x-y+n) p(y) d y= \\
& =\sum_{n=0}^{\infty} \exp (-2 \pi i l n) \int_{n}^{n+1} \exp (2 \pi i l x) d x \int_{0}^{1} f(x-y) p(y) d y= \\
& =c_{l}[p] \int_{0}^{\infty} \exp (2 \pi i l x) f(x) d x=f_{l} c_{l}[p],
\end{aligned}
$$

где мы использовали (3.3) и условие $f(x)=0$ для $x<0$. В результате получаем уравнение

$$
c_{l}[G p]=f_{l} c_{l}[p]
$$

Так как $f(x)$ неотрицательна и непрерывна, то $f_{l}<1$ для $l \neq 0$. Подставляя в полученное уравнение $p=g_{\infty}$, где $g_{\infty}$ - неподвижная точка в функциональном пространстве, т.е. $G g_{\infty}=g_{\infty}$, мы имеем $c_{l}\left[g_{\infty}\right]=0$ для $l \neq 0$ и поэтому $g_{\infty} \equiv 1$ почти всюду. Кроме того, получаем

$$
c_{l}(N)=c_{l}\left[G^{N} p\right]=f_{l}^{N} c_{l}[p],
$$

применяя уравнение (3.6) рекуррентно. Отсюда следует, что $c_{l}(N) \rightarrow 0$ при $N \rightarrow \infty$, т.е. последовательность $G^{N} p$ слабо сходится к $\int_{0}^{1} p(x) d x=$ const.

Интегральный оператор $G$ отображает $C(0,1)$ в $C(0,1)$, потому что он имеет кусочно-непрерывное ядро с разрывом на линии $x=y$ и, следовательно, может быть представлен как сумма двух интегральных операторов Вольтерра, каждый из которых отображает $C(0,1)$ в $C(0,1)$. Эти операторы имеют ядра $\{g(x-y), y<x ; 0, y \geq x\}$ и $\{0, y<x ; g(1+x-y), y \geq x\}$. Оператор $G$ компактен в $L_{2}[0,1]$-топологии, т.к. является оператором Гильберта-Шмидта [14], т.е.

$$
\operatorname{Sp} G G^{+}=\int_{0}^{1} g^{2}(x, y) d x d y=\|g\|_{L_{2}}^{2}<\infty .
$$

Спектр оператора дискретен на комплексной плоскости, за исключением точки 0. Так как $G$ имеет стохастическое ядро

$$
\int_{0}^{1} g(x, y) d x=1
$$

то его собственные значения лежат в единичном круге. Вследствие слабой сходимости $G$ имеет единственное собственное значение, равное 1 , на единичной окружности. Поэтому сходимость последовательности $G^{N} p$ является сильной в $L_{2}[0,1]$-топологии. Более того, т.к. оператор $G^{2}$ имеет непрерывное ядро на линии $x=y$, что подтверждается прямым вычислением, то он является компактным в $C(0,1)$-топологии. Поэтому последовательность $G^{N} p$ сходится на самом деле в равномерной топологии. На последнем шаге доказательства положим $p(x)=g\left(x, y_{1}\right), y_{1}=\epsilon_{1} / \hbar \omega$ 
На основе доказанной теоремы мы можем утверждать, что расстояния между дробными частями стремятся к нулю с вероятностью единица при $N \rightarrow \infty$, т.е. дробные части квазиэнергий равномерно покрывают интервал $[0, \hbar \omega]$. Поэтому статистическое описание расстояний между ними в пределе $N \rightarrow \infty$ должно быть переформулировано так, чтобы имелся нетривиальный предельный закон. С этой целью мы найдем обшее выражение для функции распределения вероятности расстояний между дробными частями.

Пусть $y$ - некоторая выбранная дробная часть квазиэнергии $\epsilon, y=(\epsilon / \hbar \omega) \bmod 1$. Обозначим символом $P_{N}(a \mid y)$ функцию распределения условной вероятности случайной величины, которая является расстоянием между точкой $y$ и ближайшей к ней справа дробной частью при условии, что марковская цепь $\left\{\zeta_{1}, \zeta_{2}, \ldots, \zeta_{N}\right\}$ попала в точку $y$. Условная вероятность того, что ближайшая к $y$ справа дробная часть превосходит $a$, определяется формулой

$$
P_{N}(a \mid y)=\lim _{b \rightarrow 0+} \mathbf{P}_{N}(a ; y, b) / \mathbf{P}_{N}(y, b)
$$

где $\mathbf{P}_{N}(y, b)$ - вероятность случайного события, для которого существует шаг $s$ такой, что $\zeta_{s} \in(y-b, y], b>0$, и $\mathbf{P}_{N}(a ; y, b)$ - вероятность случайного события, для которого $\zeta_{n} \notin(y, y+a)$ при $n=1,2, \ldots, N$ и сушествует шаг $s$ с указанным выше свойством. Вьполняются следуюшие вероятностные соотношения:

$$
\begin{aligned}
\mathbf{P}_{N}(y, b)= & 1-\mathbb{P}\left\{\zeta_{n} \notin(y-b, y], n=1, \ldots, N\right\}, \\
\mathbf{P}_{N}(a ; y, b)= & \mathbb{P}\left\{\zeta_{n} \notin(y, y+a], n=1, \ldots, N\right\}- \\
& -\mathbb{P}\left\{\zeta_{n} \notin(y-b, y+a], n=1, \ldots, N\right\} .
\end{aligned}
$$

Теперь необходимо выразить условную плотность (3.7) в терминах функции $g$. Введем операторную функцию $G_{a}(y)$, определяемую формулой

$$
\left(G_{a}(y) p\right)(x)=\int_{\gamma(a, y)} g(x, z) p(z) d z
$$

где $p(z)$ - непрерывная плотность на $[0,1]$ и $\gamma(a, y)=\{[0, y) \cup[y+a, 1)$, если $(y+a)<1$; $[y+a-1, y)$, если $(a+y)>1\}$. Тогда введенные вероятности определяются формулой

$$
\begin{aligned}
\mathbf{P}_{N}(y-b, b+a) & \equiv \mathbb{P}\left\{\zeta_{n} \notin(y-b, y+a], n=1, \ldots, N\right\}= \\
& =\int_{\gamma(a+b, y-b)}\left(G_{a+b}^{N}(y-b)\right)\left(x, y_{1}\right) d x
\end{aligned}
$$

и аналогичными формулами, получаюшимися из (3.10) при $b=0$ либо при $a=0$. Удобно избавиться от произвольности выбора точки $y$ в определении оператора $G_{a}(y)$. Для этого мы используем выражение (3.5). Сдвигаем область интегрирования в уравнении $(3.9)$ на величину $(-y)$, если $(a+y)<1$, и на величину $(1-y)$, если $(a+y)>1$. В результате имеем формулу

$$
\left(G_{a}(y) p\right)(x)=\left(G_{a} \vartheta_{y} p\right)(x-y)
$$


которая выполняется, если функция $p(z)$ периодически продолжена с единичным периодом, а $\vartheta_{y}$ есть оператор сдвига $\left(\vartheta_{y} p\right)(x)=p(x+y)$. Индукцией по $N$ получаем в качестве следствия

$$
\left(G_{a}^{N}(y) p\right)(x)=\left(G_{a}^{N} \vartheta_{y} p\right)(x-y)
$$

Поэтому формула

$$
\mathbf{P}_{N}(y-b, b+a)=\int_{a+b}^{1}\left(G_{a+b}^{N}\right)\left(x, y_{1}+b-y\right) d x
$$

имеет место для $(a+b)<1$. Сдвигая каждый интеграл на $(-b)$ и используя определение (3.5), последнее выражение удобно представить в следующей форме:

$$
\mathbf{P}_{N}(y-b, a+b)=\int_{a}^{1-b}\left(G_{a, b}^{N}\right)\left(x, y_{1}-y\right) d x
$$

где

$$
\left(G_{a, b} p\right)(x)=\int_{a}^{1-b} g(x, z) p(z) d z .
$$

Вероятность $\mathbf{P}_{N}(y-b, a+b)$, представленная в виде (3.11), явным образом дифференцируема по $b$, и можно непосредственно вычислить $P_{N}(a \mid y)$ методом Лопиталя. Суммируем полученный результат в виде отдельной теоремы.

Теорема 2. Вероятность $P_{N}(a \mid y)$ определяется формулами (3.7)-(3.8), (3.10)$(3.11)$

\section{4. АСИМПТОТИЧЕСКАЯ ФОРМУЛА}

В этом разделе мы докажем важное утверждение относительно асимптотического поведения вероятности $P_{N}(a \mid y)$ при $N \rightarrow \infty$. Введем следуюшие обозначения. Каждый оператор $G_{a}$ имеет полный набор собственных векторов с соответствующими собственными значениями $\beta_{k}(a), k=0,1,2, \ldots$, т.к. оператор $G_{a}$ компактен для каждого $a$. Только конечное множество собственных значений лежит вне произвольной окружности с центром в нуле [14]. Мы полагаем, что собственные значения для каждого а упорядочены по убыванию их модулей (если имеются собственные значения с совпадаюшими модулями, то они располагаются в порядке возрастания фазы). Порядок определяет функции $\beta(a) \equiv \beta_{0}(a), \beta_{1}(a), \beta_{2}(a)$. Эти функции дифференцируемы (см. приложение А), и по определению имеют место следуюшие неравенства: $|\beta(a)| \geq\left|\beta_{1}(a)\right| \geq$ $\left|\beta_{2}(a)\right| \geq \ldots$ Разложение

$$
G_{a}=\beta(a) I(a)+\widetilde{G}_{a}, \quad \widetilde{G}_{a} \equiv \sum_{k=1}^{\infty} \beta_{k}(a) I_{k}(a)
$$

выполняется по биортогональному набору [14], где $I_{k}(a), k=0,1, \ldots,-$ одномерные неортогональные проекторы, соответствуюшие $\beta_{k}(a), I_{0}(a) \equiv I(a)$, т.е. имеют место следующие соотношения: $I_{k}(a) I_{j}(a)=\delta_{k j} I_{k}(a),\left\|I_{k}(a)\right\|=1 ; k, j=0,1,2, \ldots$, $\sum_{k=0}^{\infty} I_{k}(a)=1$.

Искомую асимптотику определяет 
ТЕОРемА 3. Формула

$$
\mathbf{P}_{N}(a, y) \sim \beta^{N-2}(a) R(a)
$$

справедлива при $N \rightarrow \infty$ для непрерывной плотности $f()$, əде

$$
R(a)=\left(I(a) g\left(., y_{1}-y\right)\right)(1) \int_{a}^{1}(I(a) g)(x) d x .
$$

Асимптотика (4.2) равномерна по величине а при достаточно мальх ее значениях.

ДокАЗАтЕльство. А. Прежде всего мы найдем оценку нормы оператора $\widetilde{G}_{a}^{N}$. Из (4.1) следует, что

$$
\begin{aligned}
G_{a}^{N} & =\beta^{N}(a) I(a)+\widetilde{G}_{a}^{N}, \\
\widetilde{G}_{a}^{N} & =\sum_{k=1}^{\infty} \beta_{k}^{N}(a) I_{k}(a) .
\end{aligned}
$$

Мы имеем, во-первых, тривиальную оценку

$$
\left\|G-G_{a}\right\|=\max _{x \in[0,1]} \int_{0}^{a}|g(x, y)| d y \leq a\|g\|,
$$

где норма $\|. \mid\|$ понимается как норма в пространстве $C(0,1)$. Во-вторых, мы можем сконструировать конечномерную аппроксимацию $G_{0}^{(M)}$ для оператора $G$, т.е. найдем число $M$ такое, что разность между $G$ и $G_{0}^{(M)}$ имеет норму, меншшую чем $\delta<1$, где

$$
G_{a}^{(M)}=\sum_{k=0}^{M} \beta_{k}(a) I_{k}(a)
$$

Это может быть сделано благодаря компактности $G$. В-третьих, мы используем непрерывность по $a$ набора функций $\beta_{k}(a)$ (приложение А) и операторных функций $I_{k}(a)$ (приложение Б), $k=0,1, \ldots, M$, для достаточно малых значений $a$, т.е. сушествует $a^{*}(\delta, M)$, для которого $\left\|G_{0}^{(M)}-G_{a}^{(M)}\right\|<\delta$, как только $a<a^{*}$. Представляя теперь $\widetilde{G}_{a}^{N}$ в виде

$$
\widetilde{G}_{a}^{N}=\left(G_{a}^{(M)}-\beta(a) I(a)\right)^{N}+\left(G_{a}-G_{a}^{(M)}\right)^{N},
$$

используя соотношения $(4.3),(4.4)$ и оценку

$$
\left\|G_{a}-G_{a}^{(M)}\right\| \leq\left\|G_{a}-G\right\|+\left\|G-G_{0}^{(M)}\right\|+\left\|G_{0}^{(M)}-G_{a}^{(M)}\right\|
$$

находим

$$
\left\|\widetilde{G}_{a}^{N}\right\| \leq \sum_{k=1}^{M}\left|\beta_{k}(a)\right|^{N}+\left(a^{*}\|g\|+2 \delta\right)^{N},
$$

т.к. $\left\|I_{k}(a)\right\|=1$. Если $a^{*}$ и $\delta$ достаточно малы, мы получаем равномерную оценку

$$
\left\|\widetilde{G}_{a}^{N}\right\|<\left|\beta_{1}(a)\right|^{N} L
$$


c некоторой постоянной $L$, т.к. $\left|\beta_{1}(a)\right| \geq \cdots \geq\left|\beta_{M}(a)\right|$.

Б. На этом шаге доказательства мы вычислим предел в выражениях (3.7):

$$
P_{N}(a \mid y)=\widetilde{\mathbf{P}}_{N}(a, y) / \widetilde{\mathbf{P}}_{N}(y),
$$

где

$$
\widetilde{\mathbf{P}}_{N}(a, y)=-(\partial / \partial b) \int_{a}^{1-b}\left(G_{a, b}^{N-1} g\left(., y_{1}-y\right)\right)_{b=0}(x) d x
$$

и $\widetilde{\mathbf{P}}_{N}(y)=\widetilde{\mathbf{P}}_{N}(0, y)$. Необходимо заметить, что предел не может быть вычислен путем подстановки асимптотик функций $\mathbf{P}_{N}(y-b, a+b), \mathbf{P}_{N}(y, a), \mathbf{P}_{N}(y-b, b)$ в $(3.7)$ вследствие неравномерности их по $b$. Используя формулу дифференцирования

$$
\left[\left(\partial G_{a, b} / \partial b\right)_{b=0} p\right](x)=-g(x) p(1)
$$

для произвольной функции $p(x) \in C(0,1)$, мы получаем явное выражение для функции (4.8)

$$
\widetilde{\mathbf{P}}_{N}(a, y)=\left(G_{a}^{N-1} g\left(., y_{1}-y\right)\right)(1)+\sum_{s=0}^{N-2}\left(G_{a}^{N-2-s} g\left(., y_{1}-y\right)\right)(1) \int_{a}^{1}\left(G_{a}^{s} g\right)(x) d x
$$

В. Наконец, найдем асимптотику функции $\widetilde{\mathbf{P}}_{N}(a, y)$. Для того чтобы вычислить главный член асимптотики, мы пренебрегаем далее членами, имеющими порядок $N \beta^{N}(a) o(1)$, где $o(1)$ понимается как функция с двойным пределом, равным нулю при $N \rightarrow \infty, a \rightarrow+0$. В этом случае асимптотика по $N$ будет равномерной относительно величины $a$. На основе этого предписания, а также ограниченности операторной функции $G_{a}$, мы можем пренебречь первым членом в $(4.9)$ и членами с $s=0,(N-2)$, как нетипичными. Далее мы используем формулу (4.3) и оценку (4.6). В соответствии с предписанием оценка (4.6) позволяет принимать во внимание только первый член в (4.3) при вычислении асимптотики правой части (4.9). Это может быть сделано на основании оценок

и

$$
\left|\sum_{s=1}^{N-3} \beta^{-s}(a) \int_{a}^{1}\left(\widetilde{G}^{s} g\right)(x) d x\right| \leq L\|g\|\left|\beta_{1}(a)\right|\left(|\beta(a)|-\left|\beta_{1}(a)\right|\right)^{-1}=O(1)
$$

$$
\left|\sum_{s=1}^{N-3}\left(\widetilde{G}_{a}^{N-2-s} g\left(., y_{1}-y\right)\right)(1) \int_{a}^{1}\left(\widetilde{G}_{a}^{s} g\right)(x) d x\right| \leq L^{2}\|g\|^{2} N\left|\beta_{1}(a)\right|^{N-2}=N \beta^{N}(a) o(1)
$$

которые равномерны по $a$ для достаточно малых значений, т.к. $\beta(a) \rightarrow 1, \beta_{1}(a) \rightarrow \beta_{1}$, $\left|\beta_{1}\right|<1$ при $a \rightarrow+0$. В результате находим асимптотическую формулу

$$
\widetilde{\mathbf{P}}_{N}(a, y)=\beta^{N}(a) N\left(R(a) / \beta^{2}(a)+o(1)\right) .
$$

Для того чтобы найти $\widetilde{\mathbf{P}}_{N}(0, y)$, заметим, что, с одной стороны, $I(a)$ есть непрерывная операторная функция и $I(a) \rightarrow I(0) \equiv I$ при $a \rightarrow+0$. Кроме того, имеем

$$
I p=\int_{0}^{1} p(x) d x
$$

для произвольной функции $p \in C(0,1)$, т.к. в этом случае выполняется $G I p=I p$, $I G p=I p$ и поэтому $I^{+}=I$ с собственным вектором $g_{\infty}=1$. С другой стороны, плотность $g$ нормирована на интервале $[0,1]$ и периодически продолжена с единичным периодом. Имеем тогда $I(a) g \rightarrow 1, I(a) g\left(., y_{1}-y\right) \rightarrow 1$ при $a \rightarrow+0$ и $R(a) \rightarrow 1$. Следовательно, мы получаем асимптотическую формулу $\widetilde{\mathbf{P}}_{N}(0, y)=N(1+o(1)), N \rightarrow \infty$. Применяя ее к (4.7) вместе с формулой (4.10), мы завершаем доказательство. 


\section{5. УНИВЕРСАЛЬНЫЙ ЗАКОН РАСПРЕДЕЛЕНИЯ}

В этом разделе мы получаем предельный закон распределения для расстояний меж ду дробными частями. Имеется очевидная возможность того, чтобы искомый закон был нетривиальным. А именно, достаточно образовать статистику случайных переменных $N \Delta_{n}, n=1, \ldots, N$. В терминах функции $P_{N}(a \mid y)$ условная вероятность случайного события $\left\{N \Delta_{n}>a ; n=1, \ldots, N\right\}$ при условии, что марковская цепь попадает в точку $y$ на некотором шаге, имеюшем номер $n \leq N$, выражается в виде $P_{N}(a / N \mid y)$. Предельная функция распределения $P_{\infty}(a)=\lim _{N \rightarrow \infty} P_{N}(a / N \mid y)$ определяется в этом случае на основе следующего утверждения.

Теорема 4. Справедлива формула

$$
P_{\infty}(a)=\exp (-a)
$$

для любой непрерывной плотности $f($.$) .$

ДокАЗАТЕльСтво. Найдем главный член асимптотики для $\beta(a)$ при $a \rightarrow+0 . \mathrm{Ha}$ основе определений $\beta(a), I(a)$ имеем

$$
\left(G_{a} I(a) g_{\infty}\right)(x)=\beta(a)\left(I(a) g_{\infty}\right)(x) .
$$

Интегрируя по $x$ от 0 до 1 обе части равенства и затем используя стохастичность ядра $g(x, y)$, находим следуюшую формулу:

$$
\beta(a)=1-a+o(1) .
$$

Здесь мы используем оценку (Б. 2) из приложения Б, благодаря которой мы заменяем операторы $I(a)$ на $I$ в обеих частях (5.2), а также то, что $I g_{\infty}=1$.

На основе асимптотической формулы (4.2) имеем $P(a / N \mid y) \sim R(a / N)[\beta(a / N)]^{N-2}$. Применяя оценку (Б. 2) и формулу (5.3), находим, что $R(a / N) \rightarrow R(0)=1$, и поэтому

$$
P_{N}(a / N \mid y) \sim(1-(a / N)+o(a / N))^{N}
$$

при $N \rightarrow \infty$. Отсюда следует утверждение теоремы.

Доказанная теорема 4 показывает, что невозможно отличить хаотический режим от регулярного, если применяется растяжение порядка $N$ расстояний $\Delta_{n}$ при статистической обработке их дробных частей с целью получения предельного закона распределения.

\section{6. РАЗЛИЧИЕ МЕЖДУ СТАТИСТИКАМИ}

В этом разделе мы рассмотрим другой путь построения предельного распределения, описываюшего статистику дробных частей при $N \rightarrow \infty$. Как следует из теоремы 3 , можно получить нетривиальную предельную функцию $Q(a)$, если положить

$$
Q(a)=\lim _{N \rightarrow \infty}\left(P_{N}(a \mid y)\right)^{1 / N}=\beta(a) .
$$

Таким образом, $Q(a)$ содержит информацию об исходной плотности $f($.$) , и это позво-$ ляет в принципе различать регулярную и хаотическую природу спектра квазиэнергий. 
$Q(a)$, очевидно, удовлетворяет естественным условиям, предъявляемым к функции распределения. Согласно определению $\beta(a)$ имеем $\beta(0)=1, \beta(1)=0, \beta(a)$ является вешественнозначной функцией, не возрастающей по $a$. Последние свойства следуют из теории Фробениуса [16] матриц с неотрицательными элементами, т.к. $\beta(a)$ является собственным значением, имеющим максимальный модуль, и $g(x, y)$ - неотрицательное ядро на $[0,1] \times[0,1]$. Кроме того, можно получить эти свойства из асимптотической формулы (4.2).

Рассмотрим теперь частный случай исходной плотности распределения $f(x)=$ $\mu \exp (-\mu x), \mu>0$, которая, как известно [1], описывает статистику энергетических расстояний в интервале энергий с регулярной эволюцией. В этом случае подстановкой $f(x)$ в (3.3) находим

$$
g(x)=(1-\exp (-\mu))^{-1} \mu \exp (-\mu x) .
$$

Спектральная задача с ядром (3.5), соответствуюшим указанной плотности, имеет явное решение, т.к. интегральное уравнение

$$
\int_{a}^{1} g(x, y) p(y) d y=\beta(a) p(x)
$$

сводится к простейшему дифференциальному уравнению. В результате находим полньй ненулевой спектр оператора $G_{a}$ в виде

$$
\beta_{k}(a)=(1-a)(1+2 \pi i k / \mu)^{-1}, \quad k \in \mathbb{Z} .
$$

Полагая $k=0$, видим, что функция распределения линейна на [0,1],

$$
Q(a)=1-a
$$

и зависимость от $\mu$ отсутствует. Тем не менее линейная функция распределения соответствует исходным плотностям $f$, которые не являются функциями обшего вида. В самом деле, если мы используем теорию возмушений для вычисления $\beta(a)$, разлагая ее в степенной ряд по переменной $a$, то все коэффициенты, соответствующие членам со степенями, большими 1 , должны быть равны нулю. (Несмотря на недифференцируемость $g(x, y)$ мы можем строить степенные ряды, используя технику правых (левых) производных, т.к. разрыв функции $g(x, y)$ сосредоточен только на линии $x=y$.) Эти условия дают бесконечную систему уравнений для плотности $f($.$) . Поэтому, если f($.$) - функция$ обшего положения, то она нелинейна. Для того чтобы проиллюстрировать это рассуждение, найдем $\beta(a)$ с точностью до $a^{2}$. Запишем следуюшие разложения:

$$
\begin{aligned}
& p(x)=1+a q(x)+a^{2} q_{1}(x)+\ldots, \\
& \beta(a)=1-a+a^{2} \varkappa+\ldots,
\end{aligned}
$$

используя (5.3). Так как собственная функция $p(x)$ согласно теории Фробениуса [16] может быть сделана неотрицательной, то можно положить $\int_{0}^{1} p(x) d x=1$. На основе такого условия нормировки имеем $\int_{0}^{1} q(x) d x=0, \int_{0}^{1} q_{1}(x) d x=0$. Подставляя разложения (6.4), (6.5) в (6.2) и приравнивая нулю сумму членов, пропорциональных $a$, получаем интегральное уравнение Фредгольма

$$
q(x)=1-g(x)+\int_{0}^{1} g(x, y) q(y) d y
$$


Аналогичный анализ членов, пропорциональных $a^{2}$, дает интегральное уравнение

$$
q_{1}(x)=[q(x)-g(x) q(0+)+\dot{a}(x) / 2-\varkappa]+\int_{0}^{1} g(x, y) q_{1}(y) d y .
$$

Мы используем обозначение $q(0+)$ в том случае, если $a \rightarrow+0$, т.к. $p(x)$ имеет разрыв в точке $a$. Уравнение (6.6) имеет решение на основе теоремы Фредгольма, т.к. член $(1-g(x))$ ортогонален собственной функции $g_{\infty}$ с собственным значение 1 . Условие разрешимости уравнения (6.7) получается интегрированием по $x \in[0,1]$. В результате находим

$$
\varkappa=(g(1)-g(0)) / 2-q(0+) .
$$

В частности, если $f($.$) - любая плотность, обладаюшая свойством f(0)=0$, то мы имеем, используя $(3.3), g(0)=g(1)$, и поэтому $\varkappa=q(0+)$. Таким образом, необходимо решить уравнение (6.6), для того чтобы вычислить $Q(a)$ с точностью до $a^{2}$. Решение находится в терминах коэффишиентов Фурье $f_{l}$. Применяя формулы (3.6) после замены $p \Rightarrow q$, получим из (6.6) уравнение для коэффициентов $c_{l}[q]$

$$
c_{l}[q]=-f_{l}+f_{l} c_{l}[q], \quad l \neq 0,
$$

где использовано $c_{l}[g]=f_{l}$. Тогда решение имеет вид

$$
q(x)=-\sum_{l \neq 0} f_{l}\left(1-f_{l}\right)^{-1} \exp (-2 \pi i x),
$$

где $\left|f_{l}\right|<1$ и нелинейность $\varkappa$ в $\beta(a)$ определяется формулой

$$
\varkappa=\sum_{l \neq 0} f_{l}\left(1-f_{l}\right)^{-1},
$$

если ряд сходится абсолютно и $f(0)=0$.

Мы изучим теперь более детально особенно важный случай, когда плотность $f$ имеет вигнеровскую форму

$$
f_{\mathrm{W}}(x)=\mu x \exp \left(-\mu x^{2} / 2\right),
$$

описываюшую статистику энергетических расстояний в хаотической энергетической области [1]. Так как $f_{\mathrm{W}}(0)=0$, то реализуется (6.8). Абсолютная сходимость ряда следует из асимптотического выражения для $f_{l}$ при $l \rightarrow \infty$

$$
f_{l}=-(2 \pi l)^{-2}(\mu / 2)^{1 / 2} .
$$

Важно определить знак величины $\varkappa$. Асимптотика (6.9) выполняется равномерно по $l$ при $\mu \rightarrow+0$. Следовательно, мы получаем

$$
\varkappa \sim \sum_{l \neq 0} f_{l} \sim-(1 / 12)(\mu / 2)^{1 / 2},
$$




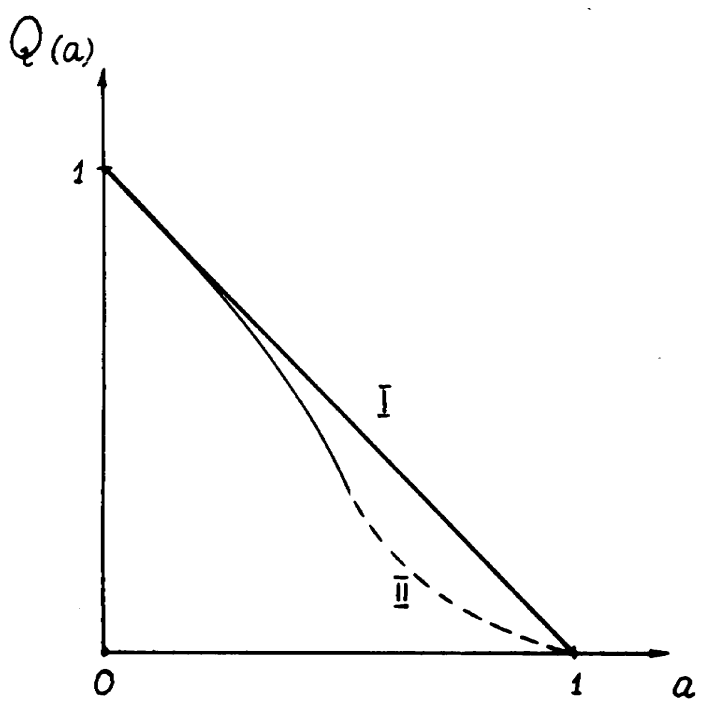

Функции распределения: I - для экспоненциальной $f$-плотности, II - для вигнеровской $f$-плотности.

и можно ожидать, что этот знак является таким же вплоть до $\mu=1$. Тогда функция распределения вероятности для этого случая имеет схематический вид, показанньй на рисунке.

\section{7. ЗАКЛЮЧЕНИЕ}

Гамильтоновы системы, периодически изменяющиеся во времени, являются удобным объектом для исследования с точки зрения детерминированного хаоса. С одной стороны, они сохраняют многие черты эволюции автономных систем, но, с другой стороны, явление хаоса проявляется для них уже в случае одной степени свободы. Если мы рассматриваем квантовые системы, для которых соответствующие классические обладают хаотическим режимом, то такие квантовые системы чувствуют наличие этого режима в квазиклассической области параметров (формально $\hbar \rightarrow 0$ ). Это явление известно как квантовый хаос. Спектры автономных квантовых систем проявляют универсальные стохастические свойства. Естественно предположить, что системы, периодически изменяющиеся во времени, также обладают аналогичными спектральными свойствами, если спектры квазиэнергий дискретны. Однако даже дискретные спектры квазиэнергий могут быть настолько сложными, что соответствующие мультипликаторы плотно покрывают единичную окружность. В связи с этим возникают дополнительные трудности, когда выполняется статистическое исследование периодически изменяющихся систем. Метод набора статистических данных, предложенный в настоящей работе, избегает этих трудностей. Он особенно эффективен, когда среднее расстояние $\Delta$ между уровнями невозмушенной системы имеет порядок $\hbar \omega$. Это связано с тем, что если $\hbar \omega \gg \Delta$, то существует много квазиэнергий $\epsilon_{n}(\lambda)$ с одинаковой целой частью величины $\epsilon_{n}(\lambda) / \hbar \omega$, и, следовательно, возможно сделать выборку квазиэнергий обычным образом. В противоположном случае $\hbar \omega \ll \Delta$ мы имеем дополнительный малый параметр, и спектр квазиэнергий отличается несущественно от спектра энергий системы с 
гамильтонианом $H(0)$ (адиабатическое возмущение). Можно учесть это отклонение с помощью теории возмушений даже для немалых $\lambda$. (В последнем случае необходимо решить спектральную задачу для гамильтониана $H(0) \neq H_{\lambda=0}$. Разность между этими гамильтонианами ренормирует спектр системы $H_{\lambda=0}$.) Предложенный метод состоит в наборе статистики расстояний между дробными частями $\left(\epsilon_{n}(\lambda) / \hbar \omega\right) \bmod 1$. В этом случае можно ожидать, что статистическая функция

$$
Q_{N}(a)=[(\text { число расстояний больше } a) / N]^{1 / N}
$$

имеет нетривиальную форму при достаточно больших $N$. Для реализации этого предположения, естественно, необходимо иметь соответствуюшую предельную теорему для статистической величины $\ln Q_{N}(a)$. (Мы благодарны профессору Л.А. Пастуру, который указал нам на это обстоятельство.) Если такая теорема имеет место, то можно ожидать, что в регулярном режиме спектр квазиэнергий является экспоненциальным и поэтому функция распределения имеет линейный вид. В противоположном случае функция распределения нелинейна и имеет универсальную форму, связанную с универсальной плотностью $f_{\mathrm{W}}$. Эта функция распределения может быть получена численно (в рамках предположения о статистической независимости расстояний между квазиэнергиями).

Авторы благодарны профессору Джерзи Лукирскому и его сотрудникам за гостеприимство во Вроцлавском институте теоретической физики в сентябре 1993 г., где возник проект настояшей работы. Мы благодарны также профессору Л.А. Пастуру за дискуссии и важные замечания. Работа была написана при поддержке Международного научного фонда.

ПРИЛОЖЕНИЕ А

Здесь мы докажем свойство гладкости функции $\beta(a)$, на которое сушественно опирались в основном тексте.

УТвЕРЖДЕНИЕ. Главное собственное значение $\beta(a)$ оператора $G_{a}$ является дифференцируемой функиией для достаточно малых значений а.

ДокАЗАтЕЛьство. Операторная функция $G_{a}$ есть интегральный оператор Гильберта-Шмидта для каждого $a$. Поэтому имеется детерминант Фредгольма $\mathfrak{D}_{a}(z)$, который является целой функцией по z и определяется выражением [17]

$$
\mathfrak{D}_{a}(z)=\sum_{l=0}^{\infty}(-z)^{l} A_{l} / l !
$$

где

$$
A_{l}=\int_{a}^{1} \ldots \int_{a}^{1} \operatorname{Det}\left(g\left(x_{i}, x_{j}\right)\right)_{i, j=1, l} d x_{1} \ldots d x_{l} .
$$

Это следует из непрерывной дифференцируемости по $a$ функции $\mathfrak{D}_{a}(z)$. Ненулевые собственные значения $\beta_{k}$ определяются уравнением $\mathfrak{D}_{a}\left(\beta^{-1}\right)=0$. Тогда на основе теоремы о неявной функции мы можем рассматривать собственные значения как неявные функции $\beta_{k}(a)$ во всех точках $a$ и для всех номеров $k$, для которых

$$
\mathfrak{D}_{a, k}^{\prime} \equiv\left(\partial \mathfrak{D}_{a}(z) / \partial z\right)_{z=\beta_{k}^{-1}} \neq 0
$$


Эти функции непрерывно дифференцируемы и удовлетворяют дифференциальному уравнению

$$
\mathfrak{D}_{a, k}^{\prime} \beta_{k}^{-2}\left(\partial \beta_{k} / \partial a\right)=\partial \mathfrak{D}_{a} / \partial a .
$$

Для $k=0$ мы можем гарантировать, что $\mathfrak{D}_{a, 0}^{\prime} \neq 0$, т.к. главное собственное значение с наибольшим модулем единственно при достаточно малых значениях $a$ в связи со сходимостью $G_{a}$ к $G$ с единственным главным собственным значением, равным 1.

\section{ПРИЛОЖЕНИЕ Б}

Докажем, что любой конечный набор операторных функций $I_{k}(a)$ непрерывен в совокупности для достаточно малых значений $a$. Для простоты рассматриваем случай, когда спектр оператора $G$ простой. Тогда достаточно доказать, что $I_{k}(a)$ непрерывна для любого $k$ и для достаточно малых значений $a$, т.к. $\beta_{k}(a) \rightarrow \beta_{k}$ при $a \rightarrow+0$. Рассмотрим, например, свойство непрерьвности функции $I(a)$. Мы используем общую формулу для проекторов [15]

$$
I(a)=(2 \pi i)^{-1} \oint_{C}\left(\alpha-G_{a}\right)^{-1} d \alpha,
$$

где $C$ является замкнутым контуром, окружаюшим единицу на комплексной плоскости $\alpha$. Требуется, чтобы $C$ не содержал собственных значений $\beta_{k}(0), k=1,2, \ldots$. Так как $\beta(a) \rightarrow 1, \beta_{1}(a) \rightarrow \beta_{1}$ при $a \rightarrow+0$ и $|\beta(a)| \geq\left|\beta_{1}(a)\right| \geq\left|\beta_{2}(a)\right| \geq \ldots$, то для достаточно малых значений $a$ норма $\left\|\left(\alpha-G_{a}\right)^{-1}\right\|$ должна быть равномерно ограничена по $a$ на контуре $C[15]$, и, следовательно, формула (Б.1) имеет смысл.

Операторная функция $G_{a}$ сходится к $G$ в равномерной топологии при $a \rightarrow+0$ вследствие (4.5). Поэтому выполняется следующая оценка:

$$
\begin{aligned}
\|I-I(a)\| & \leq(2 \pi)^{-1} \oint_{C}\left\|(\alpha-G)^{-1}-\left(\alpha-G_{a}\right)^{-1}\right\| d \alpha \leq \\
& \leq(2 \pi)^{-1} \oint_{C}\left\|(\alpha-G)^{-1}\right\|\left\|\left(\alpha-G_{a}\right)^{-1}\right\|\left\|G-G_{a}\right\| d \alpha \leq \\
& \leq \text { const }\|g\| a .
\end{aligned}
$$

Благодаря этому неравенству операторная функция $I(a)$ равномерно сходится к $I$ при $a \rightarrow+0$. Можно вывести свойство непрерывности для любой функции $I_{k}(a)$ путем аналогичного рассуждения. Для этого необходимо использовать контур, окружаюший точку $\beta_{k}(0)$ и изолируюший ее от других точек спектра оператора $G$.

\section{Список литературы}

[1] F. Haake. Quantum Signatures of Chaos. Berlin: Springer, 1991.

[2] J. N. Shirley // Phys. Rev. 1965. V. B138. № 4. P. 979-987.

[3] Hideo Sambe // Phys. Rev. 1973. V. A7. №7. P. 2203-2213.

[4] Ya. B. Zel'dovich // Sov. Phys. JETP. 1967. V. 24. P. 1006.

[5] V. I. Ritus // Sov. Phys. JETP. 1967. V. 24. P. 1041.

[6] Ya. B. Zel'dovich // Sov. Phys. Usp. 1973. V. 16. P. 427.

[7] G. Casati, L. Molinari // Progr. Theor. Phys. Suppl. 1989. № 98. P. 287-322.

[8] L. Reichl. The Transition to Chaos. Berlin: Springer, 1992.

[9] O. Bohigas // In "Chaos and Quantum Physics", Les-Houches Session LII / Eds. M. Giannoni, A. Voros and J. Zinn-Justin. Amsterdam: North-Holland, 1991. 
[10] Zyczkovski // Acta Phys. Polon. 1993. V. B24. №5. P. 967-1025.

[11] A. J. Lichtenberg, M. A. Lieberman. Regular and Stochastic Motion. New York, Heidelberg, Berlin: Springer, 1983

[12] C. F. Porter. Statistical theory of spectra fluctuations. New York: Academic Press, 1965.

[13] W. Feller. An introduction to probability theory and its applications. V. II. New York: John Willey and Sons Inc., 1966.

[14] М. Рид, Б. Саймон. Методы современной математической физики. Т. 1. Функциональный анализ. М.: Мир, 1977.

[15] Т. Като. Теория возмущений линейных операторов. М.: Мир, 1972.

[16] Ф. Р. Гантмахер. Теория матриц. М.: Наука, 1967.

[17] A.Н. Колмогоров, С.В. Фомин. Элементы теории функций и функционального анализа. М.: Наука, 1972.

Институт монокристаллов

НАН Украины

Поступила в редакцию 6.VI.1995 г.

Yu. L. Bolotin, Yu.P. Virchenko

THE QUASI-ENERGY STATISTICS FOR REGULAR AND CHAOTIC REGIMES IN QUANTUM SYSTEMS WITH HAMILTONIANS PERIODIC IN TIME

Quantum mechanical systems with Hamiltonians varying periodically in time are considered. It is supposed that spectrum of Floquet operator has no absolutely continuous part, and spacings between quasienergies may be described statistically by means of a continuous density. It is shown that statistical density induced for spacings between the fractions $\bmod (\hbar \omega)$ renormalized in the suitable manner comes arbitrarily close to exponential distribution as soon as the level number is infinitely increased. The result does not depend on the original statistical law. The alternative method of statistical description of fractions is proposed. This makes it possible to distinguish between the statistical laws of the regular and chaotic regimes. 